\title{
A MAXIMUM PROBLEM OF S.-T. YAU FOR VARIATIONAL $P$-CAPACITY
}

\author{
JIE XIAO
}

\begin{abstract}
Aвstract. Through using the semidiameter (in connection to: the mean radius and surface radius) of a convex closed hypersurface in $\mathbb{R}^{n \geq 2}$ as an sharp upper bound of the variational $(1, n) \ni p$-capacity radius, this paper settles a restriction/variant of S.-T. Yau's [66, Problem 59] from the surface area to the variational $p$-capacity whose limit as $p \rightarrow 1$ actually induces the surface area.
\end{abstract}

\section{Contents}

1. Theorem and Its Corollary

1

2. Three Lemmas and Their Proofs 4

2.1. Semidiameter 4

2.2. Mean radius 6

2.3. Variational capacity radius vs surface radius 7

3. Proofs of Theorem and Its Corollary 11

References

\section{Theorem AND Its Corollary}

In his problem section of Seminar on Differential Geometry published by Princeton University Press 1982, S.-T. Yau raised the following problem (cf. [66, Page 683, Problem 59]):

Let $h$ be a real-valued function on $\mathbb{R}^{3}$. Find (reasonable) conditions on $h$ to insure that one can find a closed surface with prescribed genus in $\mathbb{R}^{3}$ whose mean curvature (or curvature) is given by $h$.

Since posed, this problem has received a lot of attention - see also: [57, 1, 67, 31, 18] for the aspect of mean curvature; [47, 48, 6, 15, 59, 60, 58, 8, 63] for the aspect of Gauss curvature; [29, 28] and their references for the aspect of curvature measure. In this paper, we study the above problem with genus zero from the perspective of the so-called variational $p$-capacity. To be more precise, it is perhaps appropriate to review Almgren's comments on the Yau's problem (see the mid part of [66, Page 683, Problem 59]):

For "suitable" $h$ one can obtain a compact smooth submanifold $\partial A$ in $\mathbb{R}^{3}$ having mean curvature $h$ by maximizing over bounded open sets $A \subset \mathbb{R}^{3}$ the quantity

$$
F(A)=\int_{A} h d \mathcal{L}^{3}-\operatorname{Area}(\partial A) .
$$

A function $h$ would be suitable, for example, in case it were continuous, bounded, and $\mathcal{L}^{3}$ summable, and $\sup F>0$. However, the relation between $h$ and the genus of the resulting extreme $\partial A$ is not clear.

2010 Mathematics Subject Classification. 53A30.

This project was in part supported by MUN's University Research Professorship (208227463102000) and NSERC of Canada. 
Note that:

- $\int_{A} h d \mathcal{L}^{3}=\int_{A} h d \mathcal{L}^{3}$ holds for the closure $\bar{A}$ of any bounded open set $A \subset \mathbb{R}^{3}$ with $\mathcal{L}^{3}(\partial A)=0$

- $\operatorname{Area}(\partial A)=\operatorname{Area}(\partial \bar{A})$ is just the variational 1-capacity of $\bar{A}$ whenever $\bar{A}$ is convex body, i.e., $\bar{A} \in \mathbb{K}^{3}$ ( cf. [39], [23] and [42, Page 149]);

- $\mathbb{K}^{n}$ comprises all elements in $\mathscr{C}^{n}$ (all compact and convex subsets of the Euclidean space $\mathbb{R}^{n \geq 2}$ ) with nonempty interior.

So, as a restriction/variant of the Yau problem (over $\mathscr{C}^{n}$ which is contained in the collection of the closures of all bounded open sets in $\mathbb{R}^{n}$ ), it seems interesting to consider the maximum problem below:

$$
\sup \left\{F_{\operatorname{pcap}}(A)=\int_{A} h d \mathcal{L}^{n}-\operatorname{pcap}(A): \quad A \in \mathscr{C}^{n}\right\} .
$$

In the above and below, $\operatorname{pcap}(E)$ is the variational $1 \leq p<n$ capacity of an arbitrary set $E \subset \mathbb{R}^{n}$ :

$$
\operatorname{pcap}(E)=\inf _{\text {open } U \supseteq E} \operatorname{pcap}(U)=\inf _{\text {open } U \supseteq E}\left(\sup _{\text {compact } K \subseteq U} \operatorname{pcap}(K)\right),
$$

where for a compact set $K \subset \mathbb{R}^{n}$ one uses

$$
\operatorname{pcap}(K)=\inf \left\{\int_{\mathbb{R}^{n}}|\nabla f|^{p} d \mathcal{L}^{n}: f \in C_{0}^{\infty}\left(\mathbb{R}^{n}\right) \& f \geq 1_{K}\right\},
$$

with $d \mathcal{L}^{n}$ denoting the usual $n$-dimensional Lebesgue measure and $1_{K}$ being the characteristic function of $K$.

According to [30, Page 32], we have

$$
\operatorname{pcap}(A)=\operatorname{pcap}(\partial A) \quad \forall \quad A \in \mathbb{K}^{n} .
$$

This, plus [39, 23] and [42, Page 149], yields

$$
1 \operatorname{cap}(A)=\operatorname{Area}(\partial A) \equiv \mathcal{H}^{n-1}(\partial A) \quad \forall \quad A \in \mathscr{C}^{n} .
$$

Physically speaking, 2cap $(A)$ of a compact set $A \subset \mathbb{R}^{3}$ expresses the total electric charge flowing into $\mathbb{R}^{3} \backslash A$ across the boundary $\partial A$ of $A$. Moreover, in accordance with ColesantiSalani's calculation in [13] we see that for $p \in(1, n)$ the capacity $\operatorname{pcap}(A)$ of $A \in \mathbb{K}^{n}$ can be determined via

$$
\operatorname{pcap}(A)=\int_{\mathbb{R}^{n} \backslash A}\left|\nabla u_{A}\right|^{p} d \mathcal{L}^{n}=\int_{\partial A}\left|\nabla u_{A}\right|^{p-1} d \mathcal{H}^{n-1},
$$

where $d \mathcal{H}^{n-1}$ represents the $(n-1)$-dimensional Hausdorff measure on $\partial A, u_{A}$ is the so-called $(1, n) \ni p$-equilibrium potential, i.e., the unique weak solution to the following boundary value problem:

$$
\left\{\begin{array}{l}
\operatorname{div}\left(|\nabla u|^{p-2} \nabla u\right)=0 \quad \text { in } \quad \mathbb{R}^{n} \backslash A ; \\
u=1 \quad \text { on } \quad \partial A \quad \& \quad u(x) \rightarrow 0 \quad \text { as } \quad|x| \rightarrow \infty
\end{array}\right.
$$

and the vector $\nabla u_{A}$ exists almost everywhere as the non-tangential limit on $\partial A$ with respect to $d \mathcal{H}^{n-1}$; see also Lewis-Nyström's [37, Theorems 3-4].

Below is the main result of this paper.

Theorem 1.1. Given $p \in(1, n), \alpha \in(0,1)$ and a nonnegative integer $k$, let $h$ be a positive, continuous, and $L^{1}$-integrable function on $\mathbb{R}^{n}$. 
(i) There is $A_{0} \in \mathscr{C}^{n}$ such that $F_{\text {pcap }}\left(A_{0}\right)=\sup _{A \in \mathscr{C}^{n}} F_{\text {pcap }}(A)$ if and only if there exists $B_{0} \in \mathscr{C}^{n}$ such that $F_{\text {pcap }}\left(B_{0}\right) \geq 0$.

(ii) Suppose $A \in \mathbb{K}^{n}$ is a maximizer of $F_{p c a p}(\cdot)$. Then such an A satisfies the variational Eikonal p-equation $(p-1)\left|\nabla u_{A}\right|^{p}=h$ in the sense of

$$
\int_{\mathbb{S}^{n-1}} \phi \mathrm{g}_{*}\left((p-1)\left|\nabla u_{A}\right|^{p} d \mathcal{H}^{n-1}\right)=\int_{\mathbb{S}^{n-1}} \phi \mathrm{g}_{*}\left(h d \mathcal{H}^{n-1}\right) \forall \phi \in C\left(\mathbb{S}^{n-1}\right),
$$

where $\mathrm{g}_{*}\left(X d \mathcal{H}^{n-1}\right)$ is the push-forward measure of a given nonnegative measure $X d \mathcal{H}^{n-1}$ via the Gauss map $\mathrm{g}$ from $\partial A$ to the unit sphere $\mathbb{S}^{n-1}$ of $\mathbb{R}^{n}$ :

$$
\mathrm{g}_{*}\left(X d \mathcal{H}^{n-1}\right)(E)=\int_{\mathrm{g}^{-1}(E)} X d \mathcal{H}^{n-1} \quad \forall \quad \text { Borel set } \quad E \subset \mathbb{S}^{n-1},
$$

with $\mathrm{g}^{-1}$ being the inverse of the Gauss map $\mathrm{g}$. In particular, if $\partial A$ is $C^{2}$ strictly convex 1 then $(p-1)\left|\nabla u_{A}\right|^{p}=h$ holds pointwisely on $\partial A$.

(iii) If $h$ is of $C^{k, \alpha}$ and $A$, with $\partial A$ being $C^{2}$ strictly convex, is a maximizer of $F_{p c a p}(\cdot)$, then $\partial A$ is of $C^{k+1, \alpha}$.

Theorem 1.1 can actually give much more information than just a generalized solution to the above Yau problem for pcap $(\cdot)$ over $\mathscr{C}^{n}$. To see this, recall two related facts. The first is:

$$
\operatorname{div}\left(|\nabla u|^{p-2} \nabla u\right)=\left|u_{v}\right|^{p-2}\left((n-1) H u_{v}+(p-1) u_{v v}\right),
$$

where $v, u_{v}, u_{v v}$, and $H$ denote the outer unit normal vector, the first-order derivative along $v$, the second-order derivative along $v$, and the mean curvature of the level surface of $u$ respectively, and so,

$$
\operatorname{div}\left(|\nabla u|^{-1} \nabla u\right)=((n-1) H)\left(\frac{u_{v}}{\left|u_{v}\right|}\right)
$$

holds at least weakly. The second is Maz'ya's isocapacitary inequality for $p \in(1, n)$ (cf. [41]):

$$
\left(\frac{\mathcal{L}^{n}(E)}{\omega_{n}}\right)^{\frac{1}{n}} \leq\left(\left(\frac{p-1}{n-p}\right)^{p-1}\left(\frac{\operatorname{pcap}(E)}{\sigma_{n-1}}\right)\right)^{\frac{1}{n-p}} \quad \forall \quad E \subset \mathbb{R}^{n}
$$

and Federer's isoperimetric inequality (cf. [20, §3.2.43]):

$$
\left(\frac{\mathcal{L}^{n}(E)}{\omega_{n}}\right)^{\frac{1}{n}} \leq\left(\frac{\mathcal{H}^{n-1}(\partial E)}{\sigma_{n-1}}\right)^{\frac{1}{n-1}} \quad \forall \quad E \in \mathbb{K}^{n} .
$$

Here and henceforth, $\omega_{n}$ and $\sigma_{n-1}=n \omega_{n}$ stand for the volume and the surface area of the unit ball of $\mathbb{R}^{n}$ respectively. Of course, the equality in $1.5 \mathrm{~d} / 1.6$ holds as $A$ is a ball. Moreover, the left hand side of $(1.5) /(1.6)$ is called the volume radius of $E$, and the right hand sides of (1.5) and (1.6) are called the variational $p$-capacity radius and the surface radius respectively.

Now, our issue is as follows - the treatment of Theorem 1.1 brings not only Corollary 1.2 - a generalized solution to a special case (i.e., genus $=0$ ) of the original Yau problem over $\mathscr{C}^{n}$, but also a new analytic approach to some related geometric problems (see e.g. Massari's papers: [44, 45]).

Corollary 1.2. Let $h \in L^{1}\left(\mathbb{R}^{n}\right)$ be positive and continuous, $k$ be a nonnegative integer, $\alpha \in$ $(0,1)$, and

$$
F_{\mathcal{H}^{n-1}}(A)=\int_{A} h d \mathcal{L}^{n}-\mathcal{H}^{n-1}(A) \quad \forall \quad A \in \mathscr{C}^{n}
$$

\footnotetext{
${ }^{1}$ This means that $\partial A$ is of class $C^{2}$ and its Gauss curvature $G(A, x)$ is positive for any $x \in \partial A$
} 
JIE XIAO

(i) There is $A_{0} \in \mathscr{C}^{n}$ such that $F_{\mathcal{H}^{n-1}}\left(A_{0}\right)=\sup _{A \in \mathscr{C}^{n}} F_{\mathcal{H}^{n-1}}(A)$ if and only if there exists $B_{0} \in \mathscr{C}^{n}$ such that $F_{\mathcal{H}^{n-1}}\left(B_{0}\right) \geq 0$.

(ii) Suppose $A \in \mathbb{K}^{n}$ is a maximizer of $F_{\mathcal{H}^{n-1}}(\cdot)$. Then there is a Borel measure $\mu_{\mathcal{H}^{n-1}, A}$ on $\mathbb{S}^{n-1}$ such that $d \mu_{\mathcal{H}^{n-1}, A}=\mathrm{g}_{*}\left(h d \mathcal{H}^{n-1}\right)$, namely,

$$
\int_{\mathbb{S}^{n-1}} \phi d \mu_{\mathcal{H}^{n-1}, A}=\int_{\mathbb{S}^{n-1}} \phi \mathrm{g}_{*}\left(h d \mathcal{H}^{n-1}\right) \forall \phi \in C\left(\mathbb{S}^{n-1}\right) .
$$

In particular, if $\partial A$ is $C^{2}$ strictly convex, then such a maximizer A satisfies the equation $h(\cdot)=H(\partial A, \cdot)$ - the mean curvature of $\partial A$.

(iii) If $h$ is of $C^{k, \alpha}$ and $A$, with $\partial A$ being $C^{2}$ strictly convex, is a maximizer of $F_{\mathcal{H}^{n-1}}(\cdot)$, then $\partial A$ is of $C^{k+2, \alpha}$.

\section{Three Lemmas and Their Proofs}

In order to prove Theorem 1.1 and Corollary 1.2 we will not only keep in mind the isocapacitary/isoperimetric inequality $(1.5) /(1.6)$ which shows that the volume radius serves as a sharp lower bound of the variational $p$-capacity radius and the surface radius, but also explore the optimal upper bounds of these two geometric quantities in terms of the semidiameter and the mean radius; see the coming-up next three lemmas. In short, under certain conditions on $A$ and its boundary $\partial A$ we will build up the following decisive radius tree for $p \in(1, n)$ :

$$
\left(\frac{\mathcal{L}^{n}(A)}{\omega_{n}}\right)^{\frac{1}{n}} \leq\left\{\begin{array}{l}
\left(\frac{\operatorname{pcap}(A)}{\left(\frac{p-1}{n-p}\right)^{1-p} \sigma_{n-1}}\right)^{\frac{1}{n-p}} \\
\left(\frac{\mathcal{H}^{n-1}(\partial A)}{\sigma_{n-1}}\right)^{\frac{1}{n-1}}
\end{array} \leq\left\{\begin{array}{l}
\frac{\operatorname{diam}(A)}{2} \\
\frac{\mathrm{b}(A)}{2},
\end{array}\right.\right.
$$

and surprisingly find that if all principal curvatures of a given $C^{2}$ boundary $\partial A$ is in the interval $[\alpha, \beta] \subset(0, \infty)$ then

$$
\left(\frac{p-1}{(n-p) \beta}\right)^{p-1}\left(\frac{\operatorname{pcap}(A)}{\sigma_{n-1}}\right) \leq \frac{\mathcal{H}^{n-1}(\partial A)}{\sigma_{n-1}} \leq\left(\frac{p-1}{(n-p) \alpha}\right)^{p-1}\left(\frac{\operatorname{pcap}(A)}{\sigma_{n-1}}\right) .
$$

2.1. Semidiameter. The isodiameter or Bieberbach's inequality (cf. [19, Page 69] and [54, Page 318]) says that the semidiameter $2^{-1} \operatorname{diam}(A)$ of $A \subset \mathbb{R}^{n}$ dominates the volume radius of $A$ :

$$
\left(\frac{\mathcal{L}^{n}(A)}{\omega_{n}}\right)^{\frac{1}{n}} \leq \frac{\operatorname{diam}(A)}{2}
$$

with equality when $A$ is a ball. Interestingly, (2.1) has been improved through the foregoing $(1.5) /(1.6)$ and the following $(2.2) /(2.3)$.

\section{Lemma 2.1.}

(i) If $p \in(1, n)$ and $A \subset \mathbb{R}^{n}$ is a connected compact set, then

$$
\left(\left(\frac{p-1}{n-p}\right)^{p-1}\left(\frac{\operatorname{pcap}(A)}{\sigma_{n-1}}\right)\right)^{\frac{1}{n-p}} \leq \frac{\operatorname{diam}(A)}{2},
$$

with equality when $A$ is a ball.

(ii) If $A \in \mathbb{K}^{n}$, then

$$
\left(\frac{\mathcal{H}^{n-1}(\partial A)}{\sigma_{n-1}}\right)^{\frac{1}{n-1}} \leq \frac{\operatorname{diam}(A)}{2}
$$


with equality when $A$ is a ball.

Proof. Obviously, equalities in (2.2) and (2.3) occur when $A$ is a ball. Note that (2.3) is the well-known Kubota inequality (cf. [35, 40]). So, it suffices to prove the remaining part of (2.2). To do so, suppose

$$
\left\{\begin{array}{l}
\operatorname{dist}(x, A)=\inf _{y \in A}|x-y| ; \\
r \mathbb{B}^{n}=\left\{x \in \mathbb{R}^{n}:|x|<r\right\} \quad \forall \quad r>0 ; \\
\overline{\mathbb{R}^{n}}=\mathbb{R}^{n} \cup\{\infty\} \\
S(A, t)=\mathcal{H}^{n-1}\left(\left\{x \in r \mathbb{B}^{n} \backslash A: \operatorname{dist}(x, A)=t\right\}\right) \quad \forall \quad t>0 .
\end{array}\right.
$$

The flat case of Gehring's Theorem 2 in [25] implies that if

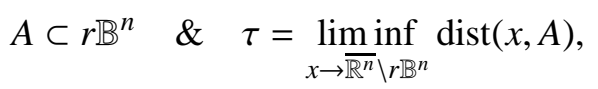

then

$$
\operatorname{pcap}\left(A, r \mathbb{B}^{n}\right) \leq\left(\int_{0}^{\tau}(S(A, t))^{\frac{1}{1-p}} d t\right)^{1-p},
$$

where

$$
\operatorname{pcap}\left(A, r \mathbb{B}^{n}\right)=\inf _{u} \int_{r \mathbb{B}^{n} \backslash A}|\nabla u|^{p} d \mathcal{L}^{n}
$$

for which the infimum ranges over all functions $u$ that are continuous in $\overline{\mathbb{R}^{n}}$ and absolutely continuous in the sense of Tonelli in $\mathbb{R}^{n}$ with $u=0$ in $A$ and $u=1$ in $\overline{\mathbb{R}^{n}} \backslash r \mathbb{B}^{n}$.

Noting such an essential fact that if $\hat{A}$ is the convex hull of $A$ then

$$
\operatorname{pcap}(A) \leq \operatorname{pcap}(\hat{A}) \quad \& \quad \operatorname{diam}(A)=\operatorname{diam}(\hat{A}),
$$

without loss of generality we may assume that $A$ is convex, and then restate Kubota's inequality (cf. [35, 27]) for such an $A$ :

$$
\frac{\mathcal{H}^{n-1}(\partial A)}{\sigma_{n-1}} \leq\left(\frac{\operatorname{diam}(A)}{2}\right)^{n-1} .
$$

This in turn implies

So, the last inequality, along with (2.4), gives

$$
\frac{S(A, t)}{\sigma_{n-1}} \leq\left(\frac{\operatorname{diam}(A)+2 t}{2}\right)^{n-1} .
$$

$$
\begin{aligned}
& \frac{\operatorname{pcap}\left(A, r \mathbb{B}^{n}\right)}{\sigma_{n-1}} \\
& \leq\left(\int_{0}^{\tau}\left(\frac{\operatorname{diam}(A)+2 t}{2}\right)^{\frac{n-1}{1-p}} d t\right)^{1-p} \\
& =\left(\left(\frac{1-p}{n-p}\right)\left(\left(\frac{\operatorname{diam}(A)}{2}+\tau\right)^{\frac{n-p}{1-p}}-\left(\frac{\operatorname{diam}(A)}{2}\right)^{\frac{n-p}{1-p}}\right)\right)^{1-p} \\
& \rightarrow\left(\left(\frac{p-1}{n-p}\right)\left(\frac{\operatorname{diam}(A)}{2}\right)^{\frac{n-p}{1-p}}\right)^{1-p} \text { as } \tau \rightarrow \infty .
\end{aligned}
$$

As a result, we get

$$
\frac{\operatorname{pcap}(A)}{\sigma_{n-1}}=\lim _{r \rightarrow \infty} \frac{\operatorname{pcap}\left(A, r \mathbb{B}^{n}\right)}{\sigma_{n-1}} \leq\left(\frac{p-1}{n-p}\right)^{1-p}\left(\frac{\operatorname{diam}(A)}{2}\right)^{n-p}
$$


whence reaching the inequality of (2.2).

2.2. Mean radius. For $A \in \mathbb{K}^{n}$, denote by (cf. [54, 1.7])

$$
h_{A}(x)=\sup _{y \in A} x \cdot y \quad \& \quad \mathrm{~b}(A)=\frac{2}{\sigma_{n-1}} \int_{\mathbb{S}^{n-1}} h_{A} d \theta
$$

the support function and the mean width of $A$ (with $d \theta$ being the standard area measure on $\mathbb{S}^{n-1}$ ) respectively, and then write $\mathrm{b}(A) / 2$ for the mean radius of $A$ according to [50]. Clearly,

$$
\frac{\mathrm{b}(A)}{2} \leq \frac{\operatorname{diam}(A)}{2}
$$

with equality when $A$ is a ball. Interestingly, the Uryasohn inequality (cf. [54. (6.25)])

$$
\left(\frac{\mathcal{L}^{n}(A)}{\omega_{n}}\right)^{\frac{1}{n}} \leq \frac{\mathrm{b}(A)}{2}
$$

holds with equality if $A$ is a ball. Even more interestingly, the forthcoming lemma reveals that (2.5) can be further improved.

\section{Lemma 2.2.}

(i) If $p \in(1, n)$ and $A \in \mathbb{K}^{n}$, then

$$
\left(\left(\frac{p-1}{n-p}\right)^{p-1}\left(\frac{\operatorname{pcap}(A)}{\sigma_{n-1}}\right)\right)^{\frac{1}{n-p}} \leq \frac{\mathrm{b}(A)}{2},
$$

with equality when $A$ is a ball.

(ii) If $A \in \mathbb{K}^{n}$, then

$$
\left(\frac{\mathcal{H}^{n-1}(\partial A)}{\sigma_{n-1}}\right)^{\frac{1}{n-1}} \leq \frac{\mathrm{b}(A)}{2}
$$

with equality when $A$ is a ball.

Proof. Since (2.7) can be seen from Chakerian's [7, (25)], it is enough to verify (2.6). Note that

$$
\frac{|x| \mathrm{b}(A)}{2}=\frac{1}{\sigma_{n-1}} \int_{\mathbb{S}^{n-1}} h_{A}(|x| \theta) d \theta
$$

is valid for any given $x \in \mathbb{R}^{n}$, and importantly, an extension of [3, Example 7.4] to $A \in \mathbb{K}^{n}$ tells us that the right side of (2.8) can be approximated by $\sum_{j=1}^{m} h_{A}\left(|x| \theta_{j}\right) \lambda_{j}$ which is the support function of $\sum_{j=1}^{m} \lambda_{j} R_{j}(A)$, where

$$
\left\{\begin{array}{l}
\lambda_{j} \in(0,1) \\
\sum_{j=1}^{m} \lambda_{j}=1 \\
R_{j}(A) \text { is an appropriate rotation of } A \text { associated to } \theta_{j} .
\end{array}\right.
$$


Therefore, by employing Colesanti-Salani's [13, Theorem 1] and by induction, we can readily obtain that if $p \in(1, n)$ then

$$
\begin{aligned}
(\operatorname{pcap}(A))^{\frac{1}{n-p}} & =\sum_{j=1}^{m} \lambda_{j}(\operatorname{pcap}(A))^{\frac{1}{n-p}} \\
& =\sum_{j=1}^{m} \lambda_{j}\left(\operatorname{pcap}\left(R_{j}(A)\right)\right)^{\frac{1}{n-p}} \\
& \leq\left(\operatorname{pcap}\left(\sum_{j=1}^{m} \lambda_{j} R_{j}(A)\right)\right)^{\frac{1}{n-p}} .
\end{aligned}
$$

Here the rotation-invariance of $\operatorname{pcap}(\cdot)$ has been used; see e.g. [19, Page 151]. Note also that the left side of (2.8) is the support function of a ball of radius $b(A) / 2$. So, a combination of the above approximation, the correspondence between a support function and a convex set, (2.9) and the well-known formula

$$
\operatorname{pcap}\left(r \mathbb{B}^{n}\right)=\sigma_{n-1}\left(\frac{p-1}{n-p}\right)^{1-p} r^{n-p},
$$

derives the left inequality of (2.6).

2.3. Variational capacity radius vs surface radius. We should point out that if $p=n-1=$ 2 then (2.6) is just Pólya's inequality [50, (5)] - here the fact that for a $C^{2}$ body $A \in \mathbb{K}^{3}$ the mean radius $\mathrm{b}(A) / 2$ is equal to $(4 \pi)^{-1}$ times the surface integral of the mean curvature has been used. To see this more transparently, let us recall that for a convex set $A$ with its boundary $\partial A$ being $C^{2}$ hypersurface,

$$
m_{j}(A, x)=\left\{\begin{array}{l}
1 \text { for } j=0 ; \\
\left(\begin{array}{c}
n-1 \\
j
\end{array}\right)^{-1} \sum_{1 \leq i_{1}<\ldots<i_{j} \leq n-1} \kappa_{i_{1}}(x) \cdots \kappa_{i_{j}}(x) \text { for } j=1, \ldots, n-1,
\end{array}\right.
$$

is the $j$-th mean curvature at $x \in \partial A$, where $\kappa_{1}(x), \ldots, \kappa_{n-1}(x)$ are the principal curvatures of $\partial A$ at the point $x$. Note that (see, e.g. [4, 22])

$$
\left\{\begin{array}{l}
m_{1}(A, x)=H(\partial A, x)=\text { mean curvature of } \partial A \text { at } x \\
m_{j}(A, x) \leq(H(\partial A, x))^{j} \text { for } \quad j=1, \ldots, n-1 \\
m_{n-1}(A, x)=G(\partial A, x)=\text { Gauss curvature of } \partial A \text { at } x .
\end{array}\right.
$$

Such a higher order mean curvature $m_{j}(A, \cdot)$ is used to produce the so-called $j$-th integral mean curvature of $\partial A$ :

$$
M_{j}(A)=\int_{\partial A} m_{j}(A, \cdot) d \mathcal{H}^{n-1}(\cdot)
$$

Clearly, we have

$$
\left\{\begin{array}{l}
M_{0}=\mathcal{H}^{n-1}(\partial A) \\
M_{1}=\int_{\partial A} H(\partial A, \cdot) d \mathcal{H}^{n-1}(\cdot) \\
M_{n-2}=\sigma_{n-1} \mathrm{~b}(K) / 2
\end{array}\right.
$$

Moreover, if $v(x)$ is the outer unit normal vector then (cf. [43])

$$
M_{0}=\int_{\partial A} x \cdot v(x) H(\partial A, x) d \mathcal{H}^{n-1}(x)
$$


if $n=2$ then the Gauss-Bonnet formula gives $M_{1}(A)=2 \pi$; and if $p=n-1=2$ then (2.6) reduces to the above-mentioned Pólya's inequality.

According to [53, (13.43)], the foregoing $S(A, t)$ has the following decomposition

$$
S(A, t)=\sum_{j=0}^{n-1}\left(\begin{array}{c}
n-1 \\
j
\end{array}\right) M_{j}(A) t^{j} .
$$

This formula is brought into (2.4) to deduce

$$
\operatorname{pcap}(A) \leq\left(\int_{0}^{\infty}\left(\int_{\partial A}(1+t H(\partial A, \cdot))^{n-1} d \mathcal{H}^{n-1}(\cdot)\right)^{\frac{1}{1-p}} d t\right)^{1-p}
$$

with equality if $A$ is a ball. Moreover, if there is a constant $\beta>0$ such that $0 \leq H(\partial A, \cdot) \leq \beta$ then (2.11) is used to derive

$$
\begin{aligned}
& \operatorname{pcap}(A) \\
& \leq\left(\int_{0}^{\infty}\left(\int_{\partial A}(1+t \beta)^{n-1} d \mathcal{H}^{n-1}(\cdot)\right)^{\frac{1}{1-p}} d t\right)^{1-p} \\
& =\left(\frac{\beta(n-p)}{p-1}\right)^{p-1} \mathcal{H}^{n-1}(\partial A) .
\end{aligned}
$$

This last estimate can be strengthened through the forthcoming radius-comparison result which partially supports the well-known Pólya-Szegö conjecture [51, 50]:

Of all convex and compact sets in $\mathbb{K}^{3}$, with a given surface area, the planar disk has the minimal electrostatic capacity $2 \operatorname{cap}(\cdot)$.

Lemma 2.3. Let $p \in(1, n)$.

(i) If there is a constant $\alpha>0$ such that $A \subset \mathbb{R}^{n}$ is $\alpha$-convex, i.e., for any $x \in \partial A$ there exists a closed ball $B$ with radius $\alpha^{-1}$ such that $x \in \partial B$ and $A \subseteq B$, then

$$
\left(\alpha^{-1}\right)^{\frac{p-1}{n-1}}\left(\left(\left(\frac{p-1}{n-p}\right)^{p-1}\left(\frac{\operatorname{pcap}(A)}{\sigma_{n-1}}\right)\right)^{\frac{1}{n-p}}\right)^{\frac{n-p}{n-1}} \geq\left(\frac{\mathcal{H}^{n-1}(\partial A)}{\sigma_{n-1}}\right)^{\frac{1}{n-1}},
$$

with equality when and only when $A$ is a ball of radius $\alpha^{-1}$.

(ii) If $A \subset \mathbb{R}^{n}$ is a connected compact set with $C^{2}$ boundary $\partial A$ and there is a constant $\beta>0$ such that $0 \leq H(\partial A, \cdot) \leq \beta$, then

$$
\left(\beta^{-1}\right)^{\frac{p-1}{n-1}}\left(\left(\left(\frac{p-1}{n-p}\right)^{p-1}\left(\frac{\operatorname{pcap}(A)}{\sigma_{n-1}}\right)\right)^{\frac{1}{n-p}}\right)^{\frac{n-p}{n-1}} \leq\left(\frac{\mathcal{H}^{n-1}(\partial A)}{\sigma_{n-1}}\right)^{\frac{1}{n-1}},
$$

with equality when and only when $A$ is a ball of radius $\beta^{-1}$.

Proof. (i) To prove (2.12), let us keep in mind the fact that if $\partial A$ is of $C^{2}$ then $A$ is $\alpha$-convex if and only if each principal curvature $\kappa_{j}$ of $\partial A$ is not less than $\alpha$, i.e., $\kappa_{j} \geq \alpha$.

Following the argument for Hurtado-Palmer-Ritoré's [32, Theorem 4.5] which is just the case $p=2$ of (2.12) we set

$$
v(x)=\phi(d(x, A)) \quad \& \quad \phi(t)=(1+\alpha t)^{\frac{p-n}{p-1}} .
$$

Then $v$ is of $C^{1,1}$ in $\mathbb{R}^{n} \backslash A$. Given $t \in(0, \infty)$. If $x \in \mathbb{R}^{n} \backslash A$ is such a point that $d(x, A)$ is twice differentiable along the line minimizing $d(x, A)$ and if

$$
A_{t}=\left\{y \in \mathbb{R}^{n}: \operatorname{dist}(y, A) \leq t\right\}
$$


then on this line one utilizes (1.4) to derive

$$
\operatorname{div}\left(|\nabla v|^{p-2} \nabla v\right)=\left|\phi^{\prime}(d(x, A))\right|^{p-2}\left((n-1) H_{t}(x) \phi^{\prime}(d(x, A))+(p-1) \phi^{\prime \prime}(d(x, A))\right)
$$

where $H_{t}$ stands for the mean curvature of the hypersurface $\partial A_{t}$ which is parallel to $\partial A$. Note that $A_{t}$ is $\left(t+\alpha^{-1}\right)^{-1}$-convex. So, one has

$$
H_{t} \geq \alpha /(1+\alpha t)
$$

at the regular points in $\partial A_{t}$. Recall that $u=u_{A}$ is the $p$-equilibrium potential. A simple calculation gives

$$
\phi^{\prime}(t)=\alpha\left(\frac{p-n}{p-1}\right)(1+t \alpha)^{\frac{1-n}{p-1}} \leq 0
$$

This, along with (2.14) and a simple computation, shows that

$$
\begin{aligned}
& \operatorname{div}\left(|\nabla v|^{p-2} \nabla v\right) \\
& =\left|\phi^{\prime}(d(x, A))\right|^{p-2} \alpha(n-1)\left(\frac{p-n}{p-1}\right)(1+d(x, A) \alpha)^{\frac{1-n}{p-1}-1}\left((1+\alpha d(x, A)) H_{t}-\alpha\right) \\
& \leq 0=\operatorname{div}\left(|\nabla u|^{p-2} \nabla u\right)
\end{aligned}
$$

holds whenever $x \mapsto d(x, A)$ is of $C^{2}$.

Next, we prove that $v \geq u$ holds in $\mathbb{R}^{n} \backslash A$. For the above given $t>0$ let $u_{t}$ and $\phi_{t}$ be the $p$-equilibrium potentials of the rings

$$
\left(A_{t}, A\right) \quad \& \quad\left(\left(t+\alpha^{-1}\right) \mathbb{B}^{n}, \alpha^{-1} \overline{\mathbb{B}^{n}}\right)
$$

respectively (cf. [36]), as well as, set $v_{t}=\phi_{t}(d(x, A))$. Then the last div-estimate, plus an integration-by-part argument, implies that

$$
\operatorname{div}\left(\left|\nabla v_{t}\right|^{p-2} \nabla v_{t}\right) \leq \operatorname{div}\left(\left|\nabla u_{t}\right|^{p-2} \nabla u_{t}\right) \quad \text { in } \quad A_{t} \backslash A
$$

is valid in the distributional sense. Now, from the weak comparison principle for $p$-Laplacian (see e.g. [55]) it follows that $v_{t} \geq u_{t}$ holds in $A_{t} \backslash A$, and so that $v \geq u$ is valid in $\mathbb{R}^{n} \backslash A$ via letting $t \rightarrow \infty$.

Note also that $\nabla u$ and $\nabla v$ have non-tangential limit $\mathcal{H}^{n-1}$-almost everywhere on $\partial A$. So, if $x \in \partial A$, then $\nabla u$ and $\nabla v$ can be defined at $x$. Upon extending $u$ and $v$ continuously to $x$ and $B$ being an exterior ball to $A$, and utilizing

$$
\left\{\begin{array}{l}
\operatorname{div}\left(|\nabla v|^{p-2} \nabla v\right) \leq \operatorname{div}\left(|\nabla u|^{p-2} \nabla u\right) \quad \text { in } \quad B \\
u(x)=v(x)=1 \text { for } \quad x \in \partial A \\
v(x) \geq u(x) \text { for } \quad x \in \mathbb{R}^{n} \backslash A \\
v-u \text { continuous on } \bar{B}
\end{array}\right.
$$

as well as taking the Hopf maximum principle into account, we get

$$
|\nabla v(x)| \leq|\nabla u(x)| \quad \forall \quad x \in \partial A .
$$


An application of (1.1) gives that

$$
\begin{aligned}
& \operatorname{pcap}(A) \\
& =\int_{\partial A}|\nabla u|^{p-1} d \mathcal{H}^{n-1} \\
& \geq \int_{\partial A}|\nabla v|^{p-1} d \mathcal{H}^{n-1} \\
& =\left(-\phi^{\prime}(0)\right)^{p-1} \mathcal{H}^{n-1}(\partial A) \\
& =\left(\left(\frac{n-p}{p-1}\right) \alpha\right)^{p-1} \mathcal{H}^{n-1}(\partial A),
\end{aligned}
$$

namely, (2.12) holds.

Of course, if $A$ is a ball with radius $\alpha^{-1}$, then equality of (2.12) trivially holds. Conversely, when equality of (2.12) is true, (2.17) is employed to derive that $|\nabla u(x)|=|\nabla v(x)|$ holds for $\mathcal{H}^{n-1}$-almost every points $x \in \partial A$. Consequently, $u=v$ holds on any exterior ball to $A$ and therefore it still true in $\mathbb{R}^{n} \backslash A$. So, the level sets of $u$ and $v$ are the same. Thanks to $u \in C^{\infty}\left(\mathbb{R}^{n} \backslash A\right)$ (cf. [13]), the level sets of $u$ are $C^{\infty}$ hypersurfaces. Since

$$
|\nabla v(x)|=\left|\phi^{\prime}(d(x, A))\right||\nabla d(x, A)| \neq 0 \quad \forall \quad x \in \mathbb{R}^{n} \backslash A,
$$

one has that $|\nabla u|=|\nabla v|$ does not vanish. Consequently,

$$
\left\{\begin{array}{l}
H_{t}=\alpha /(1+\alpha t) ; \\
\operatorname{div}\left(|\nabla v|^{p-2} \nabla v\right)=\operatorname{div}\left(|\nabla u|^{p-2} \nabla u\right) .
\end{array}\right.
$$

This in turn derives that the principal curvatures of $\partial A_{t}$ equal $\left(t+\alpha^{-1}\right)^{-1}$, and so that $\left(A_{t}\right)_{t>0}$ are concentric balls with radius $\alpha^{-1}+t$. Therefore, $A$ is a ball of radius $\alpha^{-1}$.

(ii) The general inequality $(2.13)$ can be also verified by slightly modifying the above argument for (i). The key is the selection of the function pair $(v, \phi)$ for (ii) - more precisely -

$$
v(x)=\phi(d(x, A)) \quad \& \quad \phi(t)=(1+\beta t)^{\frac{p-n}{p-1}} .
$$

Under this choice, $\alpha$, (2.14), (2.16), and (2.17) will be replaced by

$$
\left\{\begin{array}{l}
\beta \\
H_{t} \leq \beta /(1+\beta t) \\
\left\{\begin{array}{l}
\operatorname{div}\left(|\nabla v|^{p-2} \nabla v\right) \geq \operatorname{div}\left(|\nabla u|^{p-2} \nabla u\right) \quad \text { in } B \\
u(x)=v(x)=1 \quad \text { for } \quad x \in \partial A \\
v(x) \leq u(x) \text { for } \quad x \in \mathbb{R}^{n} \backslash A \\
v-u \text { continuous on } \quad \bar{B}
\end{array}\right. \\
|\nabla v(x)| \geq|\nabla u(x)| \quad \forall \quad x \in \partial A
\end{array}\right.
$$

and

$$
\begin{aligned}
& \operatorname{pcap}(A) \\
& =\int_{\partial A}|\nabla u|^{p-1} d \mathcal{H}^{n-1} \\
& \leq \int_{\partial A}|\nabla v|^{p-1} d \mathcal{H}^{n-1} \\
& =\left(\left(\frac{n-p}{p-1}\right) \beta\right)^{p-1} \mathcal{H}^{n-1}(\partial A),
\end{aligned}
$$


as desired.

The argument for equality of (2.13) is similar to that for equality of (2.6) (but this time, just using the last estimation), and so left for the interested reader.

\section{Proofs of Theorem and Its Corollary}

We are ready to prove Theorem 1.1 and its Corollary 1.2

Proof of Theorem 1.1 (i) Note that if a sequence of balls $\left(B_{j}\right)_{j \geq 1}$ in $\mathscr{C}^{n}$ tends to a single-point set then $\left(F_{\text {pcap }}\left(B_{j}\right)\right)_{j \geq 1}$ approaches zero. So, $\sup _{A \in \mathscr{C}^{n}} F_{\text {pcap }}(A)$ must be nonnegative. So, if $F_{\text {pcap }}(\cdot)$ attains its supremum at $A_{0} \in \mathscr{C}^{n}$, then $F_{\text {pcap }}\left(A_{0}\right) \geq 0$, and hence the only-if-part is verified.

To see the if-part, suppose there exists $B_{0} \in \mathscr{C}^{n}$ such that $F_{\text {pcap }}\left(B_{0}\right) \geq 0$. This, along with the hypothesis $\|h\|_{L^{1}\left(\mathbb{R}^{n}\right)}<\infty$ implies

$$
0 \leq F_{\operatorname{pcap}}\left(B_{0}\right) \leq \sup _{A \in \mathscr{C}^{n}} F_{\operatorname{pcap}}(A) \leq\|h\|_{L^{1}\left(\mathbb{R}^{n}\right)}<\infty .
$$

As a result, there is a sequence $\left(A_{j}\right)_{j \geq 1}$ in $\mathscr{C}^{n}$ such that

$$
0<F_{\operatorname{pcap}}\left(A_{j}\right) \rightarrow \sup _{A \in \mathscr{C}^{n}} F_{\mathrm{pcap}}(A) .
$$

If the inradii of $\left(A_{j}\right)_{j \geq 1}$ have no a uniform positive lower bound, then two situations should be considered. The first is that $\left(A_{j}\right)_{j \geq 1}$ collapses into a single-point set $\{a\} \in \mathscr{C}^{n}$. This situation shows the degenerate result:

$$
\sup _{A \in \mathscr{C}^{n}} F_{\text {pcap }}(A)=0=F_{\text {pcap }}(\{a\}) .
$$

The second is that $\left(A_{j}\right)_{j \geq 1}$ does not collapse into a single-point set, and consequently there is a subsequence $\left(A_{j_{k}}\right)_{k \geq 1}$ such that its inradius sequence $\left(r_{j_{k}}\right)_{k \geq 1}$ tends to zero while pcap $\left(A_{j_{k}}\right) \rightarrow$ $s \in(0, \infty]$ thanks to

$$
0 \leq F_{\text {pcap }}\left(B_{0}\right) \leq\|h\|_{L^{1}\left(\mathbb{R}^{n}\right)}-\inf _{A \in \mathscr{C}^{n}} \operatorname{pcap}(A) .
$$

Now, an application of [64, Theorem 2.1, (2.5)] (cf. [65, Theorem 3.2] for a rough constant) produces

$$
\left(\frac{\mathcal{H}^{n-1}\left(\partial A_{j_{k}}\right)}{\sigma_{n-1}}\right)^{\frac{1}{n-1}} \leq\left(\frac{p(n-1)}{n(p-1)}\right)^{\frac{p-1}{n-p}}\left(\left(\frac{p-1}{n-p}\right)^{p-1}\left(\frac{\operatorname{pcap}\left(A_{j_{k}}\right)}{\sigma_{n-1}}\right)\right)^{\frac{1}{n-p}} .
$$

So, if $\left(\mathcal{H}^{n-1}\left(\partial A_{j_{k}}\right)\right)_{k \geq 1}$ is unbounded, then $s=\infty$ and hence a contradiction occurs below:

$$
0 \leq F_{\operatorname{pcap}}\left(B_{0}\right) \leq\|h\|_{L^{1}\left(\mathbb{R}^{n}\right)}-\lim _{k t o \infty} \operatorname{pcap}\left(A_{j_{k}}\right)=-\infty<0 .
$$

On the other hand, if $\left(\mathcal{H}^{n-1}\left(\partial A_{j_{k}}\right)\right)_{k \geq 1}$ is bounded, then an application of the known Osserman inradius inequality (cf. [49, 52]) ensures

$$
\mathcal{L}^{n}\left(A_{j_{k}}\right) \leq r_{j_{k}} \mathcal{H}^{n-1}\left(\partial A_{j_{k}}\right)-(n-1) r_{j_{k}}^{2} \sqrt{\omega_{n}\left(n^{-1} \mathcal{H}^{n-1}\left(\partial A_{j_{k}}\right)\right)^{n-2}},
$$

and hence $\mathcal{L}^{n}\left(A_{j_{k}}\right) \rightarrow 0$ owing to $r_{j_{k}} \rightarrow 0$. This, plus $h \in L^{1}\left(\mathbb{R}^{n}\right)$, derives the following contradiction:

$$
0 \leq F_{\operatorname{pcap}}\left(B_{0}\right) \leq \lim _{k \rightarrow \infty} F_{\operatorname{pcap}}\left(A_{j_{k}}\right)=\lim _{k \rightarrow \infty}\left(\int_{A_{j_{k}}} h d \mathcal{L}^{n}-\operatorname{pcap}\left(A_{j_{k}}\right)\right)=-s<0 .
$$

The above analysis for $s \in(0, \infty]$ indicates that the second situation will not happen.

Thus, it remains to deal with the case that the inradii of $\left(A_{j}\right)_{j \geq 1}$ have a uniform positive lower bound $r_{0}$. Under this case, using (2.10) and (2.2) we obtain 


$$
0<r_{0}=\left(\left(\frac{p-1}{n-p}\right)^{p-1}\left(\frac{\operatorname{pcap}\left(r_{0} \mathbb{B}^{n}\right)}{\sigma_{n-1}}\right)\right)^{\frac{1}{n-p}} \leq\left(\left(\frac{p-1}{n-p}\right)^{p-1}\left(\frac{\operatorname{pcap}\left(A_{j}\right)}{\sigma_{n-1}}\right)\right)^{\frac{1}{n-p}} \leq \frac{\operatorname{diam}\left(A_{j}\right)}{2}
$$

Utilizing $h \in L^{1}\left(\mathbb{R}^{n}\right)$ again, we get

$$
F_{\text {pcap }}(A) \leq\|h\|_{L^{1}\left(\mathbb{R}^{n}\right)}-\operatorname{pcap}(A) \quad \forall \quad A \in \mathscr{C}^{n},
$$

whence discovering via (1.5)

$$
F_{\operatorname{pcap}}\left(A_{j}\right) \leq\|h\|_{L^{1}\left(\mathbb{R}^{n}\right)}-\sigma_{n-1}\left(\frac{p-1}{n-p}\right)^{1-p}\left(\frac{\mathcal{L}^{n}\left(A_{j}\right)}{\omega_{n}}\right)^{\frac{n-p}{n}} .
$$

Consequently, if $\left(\operatorname{diam}\left(A_{j}\right)\right)_{j \geq 1}$ were unbounded, then $\left(\mathcal{L}^{n}\left(A_{j}\right)\right)_{j \geq 1}$ would be unbounded due to (3.1), and hence an application of (3.2) would derive that $\left(F_{\text {pcap }}\left(A_{j}\right)\right)_{j \geq 1}$ has a subsequence approaching $-\infty-$ this is impossible thanks to

$$
\lim _{j \rightarrow \infty} F_{\text {pcap }}\left(A_{j}\right) \geq F_{\text {pcap }}\left(B_{0}\right) \geq 0 .
$$

Therefore, all diameters $\operatorname{diam}\left(A_{j}\right)$ have a uniform upper bound. Now, by 3.1 and the classic Blaschke selection principle (see e.g. [54, Theorem 1.8.6]), we can choose a subsequence of $\left(A_{j}\right)_{j \geq 1}$ that converges to a non-degenerate $A_{0} \in \mathbb{K}^{n}$. Since pcap $(\cdot)$ is continuous (cf. [42, Pages 142-143]) and $h \in C\left(\mathbb{R}^{n}\right)$ (i.e., $h$ is continuous in $\left.\mathbb{R}^{n}\right), F_{\text {pcap }}(\cdot)$ is continuous, and so, $A_{0}$ is a maximizer of $F_{\text {pcap }}(\cdot)$, i.e.,

$$
F_{\text {pcap }}\left(A_{0}\right)=\sup _{A \in \mathscr{C}^{n}} F_{\text {pcap }}(A),
$$

as desired.

(ii) For $A, B \in \mathbb{K}^{n}$ and $t \in(0,1)$ let $C_{t}=A+t B$. Then

$$
C_{t} \in \mathbb{K}^{n} \quad \& \quad h_{C_{t}}=h_{A}+t h_{B}
$$

Using Tso's variational formula for $\int_{A} h d \mathcal{L}^{n}$ in [60, (4)] and the variational formula for $\operatorname{pcap}(\cdot)$ in [12, Theorem 1.1] (see also [33, Corollary 3.16] or [34, Theorem 2.5] for 2cap $(\cdot)$ ), we obtain

$$
\left.\frac{d}{d t} F_{\mathrm{pcap}}\left(C_{t}\right)\right|_{t=0}=\int_{\partial A} h_{B}(\mathrm{~g}) h d \mathcal{H}^{n-1}-\int_{\partial A} h_{B}(\mathrm{~g})(p-1)\left|\nabla u_{A}\right|^{p} d \mathcal{H}^{n-1} .
$$

Obviously, if $A$ is a maximizer of $F_{\text {pcap }}(\cdot)$, then it must be a critical point of $F_{\text {pcap }}\left(C_{t}\right)$ and thus

$$
\left.\frac{d}{d t} F_{\operatorname{pcap}}\left(C_{t}\right)\right|_{t=0}=0
$$

This and (3.3) derive

$$
\int_{\partial A} h_{B}(\mathrm{~g})(p-1)\left|\nabla u_{A}\right|^{p} d \mathcal{H}^{n-1}=\int_{\partial A} h_{B}(\mathrm{~g}) h d \mathcal{H}^{n-1} .
$$

A combined application of (3.4) and [54, Lemmas $1.7 .9 \& 1.8 .10]$ gives that 


$$
\begin{aligned}
& \int_{\mathbb{S}^{n-1}} \phi \mathrm{g}_{*}\left((p-1)\left|\nabla u_{A}\right|^{p} d \mathcal{H}^{n-1}\right) \\
& =\int_{\partial A} \phi(\mathrm{g})(p-1)\left|\nabla u_{A}\right|^{p} d \mathcal{H}^{n-1} \\
& =\int_{\partial A} \phi(\mathrm{g}) h d \mathcal{H}^{n-1} \\
& =\int_{\mathbb{S}^{n-1}} \phi \mathrm{g}_{*}\left(h d \mathcal{H}^{n-1}\right)
\end{aligned}
$$

holds for any $\phi \in C\left(\mathbb{S}^{n-1}\right)$, and thereby reaching (1.3). Moreover, if $\partial A$ is $C^{2}$ strictly convex, then the Gauss map from $\partial A$ to $\mathbb{S}^{n-1}$ is a diffeomorphism, and hence (1.3) is equivalent to

$$
(p-1)\left|\nabla u_{A}(x)\right|^{p}=h(x) \quad \forall \quad x \in \partial A .
$$

(iii) Suppose $h \in C^{k, \alpha}$ with $k$ being a nonnegative integer. Since $\partial A$ is of $C^{2}$, an application of [38, Theorem 1] (cf. [24, 17, 56, 61, 26, 46]) yields that $u_{A} \in C^{1, \hat{\alpha}}(A)$ is valid for some $\hat{\alpha} \in(0,1)$. The last equation and $h \in C^{k, \alpha}\left(\mathbb{R}^{n}\right)$ with $\alpha \in(0,1)$ derive that

$$
\left|\nabla u_{A}\right|=\left(\frac{h}{p-1}\right)^{\frac{1}{p}}
$$

is of $C^{k, \alpha}$. Note that $\partial A$ is $C^{2}$ strictly convex. So, if $\partial A$ is represented locally as $y_{n}=$ $\psi\left(x_{1}, \ldots, x_{n-1}\right)$, then the map

$$
\left(x_{1}, \ldots, x_{n-1}\right) \mapsto \nabla u_{A}\left(x_{1}, \ldots, x_{n-1}, \psi\left(x_{1}, \ldots, x_{n-1}\right)\right)
$$

is of $C^{k, \alpha}$. Thus, a combination of the chain rule (or the implicit function theorem) and the estimate $0<\inf _{\partial A} h \leq \sup _{\partial A} h<\infty$ imply that $\psi$ is of $C^{1+k, \alpha}$. This in turn implies that $\partial A$ is of $C^{1+k, \alpha}$.

Proof of Corollary 1.2 The argument for Corollary (i) is very similar to that for Theorem 1.1 (i) except that (3.1) and (3.2) are replaced respectively by their endpoint $(p=1)$ cases:

$$
0<r_{0}=\left(\frac{\mathcal{H}^{n-1}\left(\partial\left(r_{0} \mathbb{B}^{n}\right)\right)}{\sigma_{n-1}}\right)^{\frac{1}{n-1}} \leq\left(\frac{\mathcal{H}^{n-1}\left(\partial A_{j}\right)}{\sigma_{n-1}}\right)^{\frac{1}{n-1}} \leq 2^{-1} \operatorname{diam}\left(A_{j}\right)
$$

and

$$
F_{\mathcal{H}^{n-1}}\left(A_{j}\right) \leq\|h\|_{L^{1}\left(\mathbb{R}^{n}\right)}-\sigma_{n-1}\left(\frac{\mathcal{L}^{n}\left(A_{j}\right)}{\omega_{n}}\right)^{\frac{n-1}{n}} .
$$

To reach Corollary (ii), recall that for $C_{t}=A+t B$ with $A, B \in \mathbb{K}^{n}$ and $t \in(0,1)$ (in the proof of Theorem 1.1 (ii)) there exists a curvature measure $\mu_{\mathcal{H}^{n-1}, A}$ on $\mathbb{S}^{n-1}$ such that

$$
\left\{\begin{array}{l}
\mathcal{H}^{n-1}(\partial A)=(n-1)^{-1} \int_{\mathbb{S}^{n-1}} h_{A} d \mu_{\mathcal{H}^{n-1}, A} \\
\left.\frac{d}{d t} \mathcal{H}^{n-1}\left(\partial C_{t}\right)\right|_{t=0}=\int_{\mathbb{S}^{n-1}} h_{B} d \mu_{\mathcal{H}^{n-1}, A}
\end{array}\right.
$$

Since $A$ is a maximizer of $F_{\mathcal{H}^{n-1}}(\cdot)$, it is a critical point of $F_{\mathcal{H}^{n-1}}(\cdot)$, and consequently,

whence yielding (1.7) via

$$
\left.\frac{d}{d t} F_{\mathcal{H}^{n-1}}\left(C_{t}\right)\right|_{t=0}=0
$$

$$
d \mu_{\mathcal{H}^{n-1}, A}=\mathrm{g}_{*}\left(h d \mathcal{H}^{n-1}\right) .
$$


Furthermore, if $\partial A$ is $C^{2}$ strictly convex, then the Gauss map $g: \partial A \mapsto \mathbb{S}^{n-1}$ is a diffeomorphic transformation, and hence (1.7) reduces to the mean curvature equation

$$
h(x)=H(\partial A, x) \quad \forall \quad x \in \partial A
$$

through using the variational formula for $\mathcal{H}^{n-1}$ (see e.g. [14, 11, 9, 10])

$$
\left.\frac{d}{d t} \mathcal{H}^{n-1}\left(\partial C_{t}\right)\right|_{t=0}=(n-1) \int_{\partial A} h_{B}(\mathrm{~g}) H(\partial A, \cdot) d \mathcal{H}^{n-1}(\cdot)
$$

To validate Corollary (iii), note once again that under $\partial A$ being $C^{2}$ strictly convex one has that if $A \in \mathbb{K}^{n}$ is a maximizer of $F_{\mathcal{H}^{n-1}}$ then $h(\cdot)=H(\partial A, \cdot)$ holds on $\partial A$. Also, since (cf. [16, Page 197])

$$
(n-1) H(\partial A, x)=\Delta b_{A}(x) \quad \forall \quad x \in \partial A
$$

where

$$
b_{A}=d_{A}-d_{\mathbb{R}^{n} \backslash A} \quad \& \quad d_{E}(x)=\operatorname{dist}(x, E)=\min _{y \in E}|x-y| \quad \forall \quad E \in \mathbb{K}^{n},
$$

one concludes that

$$
\Delta b_{A}(x)=(n-1) h(x) \quad \forall \quad x \in \partial A,
$$

and so $b_{A}$ is of $C^{k+2, \alpha}$ provided $h$ is of $C^{k, \alpha}$, and consequently, $\partial A$ is of $C^{k+2, \alpha}$ due to DelfourZolésio's [16, Theorem 5.5].

Remark. The previous arguments for Theorem 1.1 and its Corollary 1.2, (1.5)-(1.6), the classic variational formula for the volume, and regularities for the Monge-Ampére equations established in [1, 5, 62] can be used to obtain a natural Minkowski type property - under the hypothesis that $h \in L^{1}\left(\mathbb{R}^{n}\right)$ is positive and continuous, $k$ is a nonnegative integer, $\alpha \in(0,1)$, $\beta \in(0, \infty), \mathbb{K}_{\beta}^{n}$ consists of all elements in $\mathbb{K}^{n}$ whose inradii are not less than $\beta$, and

$$
F_{\mathcal{L}^{n}}(A)=\int_{A} h d \mathcal{L}^{n}-\mathcal{L}^{n}(A) \quad \forall \quad A \in \mathbb{K}_{\beta}^{n},
$$

one has:

- There is $A_{0} \in \mathbb{K}_{\beta}^{n}$ such that $F_{\mathcal{L}^{n}}\left(A_{0}\right)=\sup _{A \in \mathscr{C}^{n}} F_{\mathcal{L}^{n}}(A) \geq 0$ if and only if there exists $B_{0} \in \mathbb{K}_{\beta}^{n}$ such that $F_{\mathcal{L}^{n}}\left(B_{0}\right) \geq 0$.

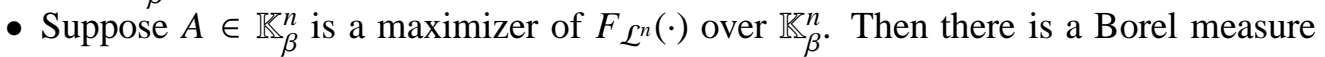
$\mu_{\mathcal{L}^{n}, A}$ on $\mathbb{S}^{n-1}$ such that $d \mu_{\mathcal{L}^{n}, A}=\mathrm{g}_{*}\left(h d \mathcal{H}^{n-1}\right)$, namely,

$$
\int_{\mathbb{S}^{n-1}} \phi d \mu_{\mathcal{L}^{n}, A}=\int_{\mathbb{S}^{n-1}} \phi \mathrm{g}_{*}\left(h d \mathcal{H}^{n-1}\right) \forall \phi \in C\left(\mathbb{S}^{n-1}\right) .
$$

In particular, if $\partial A$ is $C^{2}$ strictly convex, then such a maximizer $A$ satisfies the inverse Gauss curvature equation $h(\cdot)=(G(\partial A, \cdot))^{-1}$.

- If $h$ is of $C^{k, \alpha}$ and $A$, with $\partial A$ being $C^{2}$ strictly convex, is a maximizer of $F_{\mathcal{L}^{n}}(\cdot)$ over $\mathbb{K}_{\beta}^{n}$, then $\partial A$ is of $C^{k+2, \alpha}$.

Acknowledgement. The author is grateful to the referee for his/her useful comment on the proof of Theorem 1.1(i): $\mathcal{H}^{n-1}\left(\partial A_{j_{k}}\right) \rightarrow \infty$ can be also obtained by applying the BonessenFuglede inequality in [2, 21] to get that if $r_{j_{k}} \rightarrow 0$, then the corresponding circumradius tends to infinity due to $\mathcal{L}^{n}\left(A_{j_{k}}\right) \rightarrow 0$ and hence the isoperimetric deficit approaches infinity. 


\section{REFERENCES}

[1] I. Ja. Bakel'man and B. E. Kantor, Estimates of the solutions of quasilinear elliptic equations that are connected with problems of geometry "in the large", Mat. Sb. (N.S.) 91(133)(1973)336-349,471.

[2] T. Bonnesen, Über die sioperimetrische Defizit ebener Figuren, Math. Ann. 91(1924)252-268.

[3] C. Borell, Hitting probability of killed Brownian motion: A study on geometric regularity, Ann. Sci. Ecole Norm. Supér. Paris 17(1984)451-467.

[4] H. L. Bray and P. Miao, On the capacity of surfaces in manifolds with nonnegative scalar curvature, Invent. Math. 172(2008)459-475.

[5] L. Caffarelli, Interior a priori estimates for solutions of fully non-linear equations, Ann. Math. 131(1989)189-213.

[6] L. Caffarelli, L. Nirenberg and J. Spruck, Nonlinear second order elliptic. IV. Starshaped compact Weingarten hypersufaces, in: Ohya, Y. et al. (eds) Current Topic in PDE's, pp.1-26 Tolyo: Kinokumize 1988.

[7] G. D. Chakerian, Isoperimetric inequalities for the mean width of a convex body, Geometriae Ded. 1(1973)356-362.

[8] K.-S. Chou and X.-J. Wang, A logarithmic Gauss curvature flow and the Minkowski problem, Ann. Inst. Henri Poincaré, Analyse non linéaire 17,6(2000)733-751.

[9] A. Colesanti, Brunn-Minkowski inequalities for variational functionals and related problems, Adv. Math. 194(2005)105-140.

[10] A. Colesanti, From the Brunn-Minkowski inequality to a class of Poincaré type inequalities, Commun. Contemp. Math. 10(2008)765-772.

[11] A. Colesanti and E. S. Gómez, Functional inequalities derived from the Brunn-Minkowski inequalities for quermassintegrals, J. Convex Anal. 17(2010)35-49.

[12] A. Colesanti, K. Nyström, P. Salani, J. Xiao, D. Yang and G. Zhang, The Hadamard variational formula and the Minkowski problem for p-capacity, Adv. Math. 285(2015)1511-1588.

[13] A. Colesanti and P. Salani, The Brunn-Minkowski inequality for p-capacity of convex bodies, Math. Ann. 327(2003)459-479.

[14] G. Crasta, I. Fragalá and F. Gazzola, On a long-standing conjecture by Pólya-Szegö and related topics, Z. Angew. Math. Phys. 56(2005)763-782.

[15] P. Delanoë, Plongements raiaux $S^{n} \rightarrow R^{n+1}$ a courbure de Gauss positive prescrite, Ann. Sci. Éc. Norm. Sup'er. 18(1988)635-649.

[16] M. C. Delfour and J.-P. Zolésio, Shape analysis via oriented distance functions, J. Funct. Anal. 123(1994)129-201.

[17] E. DiBenendetto, $C^{1, \alpha}$ local regularity of weak solutions of degenerate elliptic equations, Nonlinear Anal. 7(1983)827-850.

[18] M. Eichmair, The Plateau problem for marginally outer trapped surfaces, J. Differential Geom. 83(2009)551-583.

[19] L. C. Evans and R. F. Gariepy, Measure Theory and Fine Properties of Functions, CRC Press LLC, 1992.

[20] H. Federer, Geometric Measure Theory, Springer-Verlag, 1969.

[21] B. Fuglede, Stability in the isoperimetric problem for convex or nearly spherical domains in $\mathbf{R}^{\mathbf{n}}$, Trans. Amer. Math. Soc. 314(1989)619-638.

[22] A. Freire and F. Schwartz, Mass-capacity inequalities for conformally flat manifolds with boundary, Comm. Partial Differential Equations 39(2014)98-119.

[23] R. J. Gardner and D. Hartenstine, Capacities, surface area, and radial sums, Adv. Math. 221(2009)601-626.

[24] N. Garofalo and E. Sartori, Symmetry in exterior boundary value problems for quasilinear elliptic equations via blow-up and a priori estimates, Adv. Differential Equations 4(1999)137-161.

[25] F. W. Gehring, Inequalities for condensers, hyperbolic capacity, and extremal lengths, Michigan Math. J. 18(1971)1-20.

[26] D. Gilbarg and N. Trudinger, Elliptic Partial Differential Equations of Second Order, 2nd edition, SpringerVerlag, 1983.

[27] P. Gritzmann, A characterization of all loglinear inequalities for three quermassintegrals of convex bodies, Proc. Amer. Math. Soc. 104(1988)563-570.

[28] P. Guan, J. Li and Y. Li, Hypersurfaces of prescribed curvature measures, Duke Math. J. 161(2012)19271942.

[29] P. Guan, C. Lin and X.-N. Ma, The existence of convex body with prescribed curvature measures, IMRN, 2009(2009)1947-1975. 
[30] J. Heinonen, T. Kilpeläinen and O. Martio, Nonlinear Potential Theory of Degenerate Elliptic Equations, Dover Publications, Inc., Mineola, New York, 2006.

[31] Y.-J. Hsu, S.-J. Shiau and T.-H. Wang, Graphs with prescribed mean curvature in the sphere, Bull. Inst. Math. Academia Sin. 28:4(2000)215-223.

[32] A. Hurtado, V. Palmer and M. Ritoré, Comparison results for capacity, Indiana Univ. Math. J. 61(2012)539555.

[33] D. Jerison, A Minkowski problem for electrostatic capacity, Acta Math. 176(1996)1-47.

[34] D. Jerison, The direct method in the calculus of variations for convex bodies, Adv. Math. 122(1996)262-279.

[35] T. Kubota, Über die konvex-geschlossenen Mannigfaltigkeiten im n-dimensionalen Raume, Sci. Rep. Tojoku Univ. 14(1925)85-99.

[36] J. Lewis, Capacitary functions in convex rings, Arch. Rational Mech. Anal. 66(1977)201-224.

[37] J. Lewis and K. Nyström, Regularity and free boundary regularity for the p-Laplacian in Lipschitz and $C^{1}$-domains, Ann. Acad. Sci. Fenn. Math. 33(2008)523-548.

[38] G. M. Lieberman, Boundary regularity for solutions of degenerate elliptic equations, Nonlinear Anal. 12(1988)1203-1219.

[39] M. Ludwig, J. Xiao and G. Zhang, Sharp convex Lorentz-Sobolev inequalities, Math. Ann. 350(2011)169197.

[40] F. Maggi, M. Ponsiglione and A. Pratelli, Quantitative stability in the isodiametric inequality via the isoperimetric inequality, Trans. Amer. Math. Soc. 366(2014)1141-1160.

[41] V. Maz'ya, Conductor and capacitary inequalities for functions on topological spaces and their applications to Sobolev-type imbeddings, J. Funct. Anal. 224(2005)408-430.

[42] V. Maz'ya, Sobolev Spaces with applications to elliptic partial differential equations, Springer-Verlag Berlin Heidelberg, 2011.

[43] O. Mendez and W. Reichel, Electrostatic characterization of spheres, Forum Math. 12(2000)223-245.

[44] U. Massari, Esistenza e regolaritá delle ipersuperfice di curvatura media assegnata in $R^{n}$, Arch. Rational Mech. Anal. 55 (1974)357-382.

[45] U. Massari, Frontiere orientate di curvatura media assegnata in $L_{p}$, Rend. Sem. Mat. Univ. Padova 53 (1975)37-52.

[46] L. Mou and P. Yang, Regularity for n-harmonic maps, J. Geom. Anal. 6(1996)91-112.

[47] V. I. Oliker, Hypersurfaces in $R^{n+1}$ with prescribed Gaussian curvature and related equations of MongeAmpère type, Comm. Partial Diff. Equ. 9(1984)807-839.

[48] V. I. Oliker, The problem of embedding $S^{n}$ into $R^{n+1}$ with prescribed Gauss curvature and its solution by variational methods, Trans. Amer. Math. Soc. 295(1986)291-303.

[49] R. Osserman, Bonnesen-stype isoperimetric inequalities, Amer. Math. Monthly 86(1979)1-29.

[50] G. Pólya, Estimating electrostatic capacity, Amer. Math. Monthly 54(1947)201-206.

[51] G. Pólya and G. Szegö, Inequalities for the capacity of a condenser, Amer. J. Math. 67(1945)1-32.

[52] J.R. Sangwine-Yager, A Nonnesen-style inradius inequality in 3-space, Pacific J. Math. 134(1988)173-178.

[53] L. Santaló, Integral Geometry and Geometric Probability, Encyclopedia of Mathematics and Its Applications, Vol 1, Addison-Wesley, 1976.

[54] R. Schneider, Convex Bodies: The Brunn-Minkowski Theory, Cambridge Univ. Press, 1993.

[55] P. Tolksdorf, On the Dirichlet problem for quasilinear elliptic equations in domains with conical boundary points, Comm. Part. Diff. Equa. 8(1983)773-817.

[56] P. Tolksdorf, Regularity for a more general class of quasilinear elliptic equations, J. Diff. Equa. 51(1984)126-150.

[57] A. E. Treibergs and W. Wei, Embedded hyperspheres with prescribed mean curvature, J. Differential Geom. 18(1983)513-521.

[58] K. S. Tso, Deforming a hypersurface by its Gauss-Kronecker curvature, Comm. Pure Appl. Math. 38(1985)867-882.

[59] K. S. Tso, Convex hypersurfaces with prescribed Gauss-Kronecker curvature, J. Differential Geom. 34(1991)389-410.

[60] K. S. Tso, A direct method approach for the existence of convex hypersurfaces with prescribed GaussKronecker curvature, Math. Z. 209(1992)339-334.

[61] K. Uhlenbeck, Regularity of a class of nonlinear elliptic systems, Acta Math. 138(1970)219-240.

[62] J. I. E. Urbas, Regularity of generalized solutions of Monge-Ampére equations, Math. Z. 197(1988)365-393.

[63] X.-J. Wang, Existence of convex hypersurfaces with prescribed Gauss-Kronecker curvature, Trans. Amer. Math. Soc. 348(1996)4501-4524

[64] J. Xiao, P-capacity vs surface-area, arXiv:1506.03827v2[math.DG]15June2015. 
[65] X. Xu, Some results on functional capacity and their applications to p-Laplacian problems involving measure data, Nonlinear Anal. TMA 27(1996)17-36.

[66] S.-T. Yau, Problem section. In: S.-T. Yau (ed.) Seminar on Differential Geometry, 669-706, Ann. Math. Stud. 102, Princeton University Press, Princeton, N.J., 1982.

[67] S.-T. Yau, A remark on the existence of sphere with prescribed mean curvature, Asian J. Math. 1:2(1997)293-294.

Department of Mathematics and Statistics, Memorial University, St. John's, NL A1C 5S7, Canada

E-mail address: jxiao@mun.ca 